\title{
Metagenomics analysis of microbial communities associated with a traditional rice wine starter culture (Xaj-pitha) of Assam, India
}

\author{
Sudipta Sankar Bora ${ }^{1} \cdot$ Jyotshna Keot $^{1} \cdot$ Saurav Das $^{1} \cdot$ Kishore Sarma $^{1} \cdot$ \\ Madhumita Barooah ${ }^{1}$
}

Received: 22 April 2016/ Accepted: 5 July 2016/Published online: 15 July 2016

(c) The Author(s) 2016. This article is published with open access at Springerlink.com

\begin{abstract}
This is the first report on the microbial diversity of xaj-pitha, a rice wine fermentation starter culture through a metagenomics approach involving Illuminebased whole genome shotgun (WGS) sequencing method. Metagenomic DNA was extracted from rice wine starter culture concocted by Ahom community of Assam and analyzed using a MiSeq ${ }^{\circledR}$ System. A total of 2,78,231 contigs, with an average read length of $640.13 \mathrm{bp}$, were obtained. Data obtained from the use of several taxonomic profiling tools were compared with previously reported microbial diversity studies through the culture-dependent and culture-independent method. The microbial community revealed the existence of amylase producers, such as Rhizopus delemar, Mucor circinelloides, and Aspergillus sp. Ethanol producers viz., Meyerozyma guilliermondii, Wickerhamomyces ciferrii, Saccharomyces cerevisiae, Candida glabrata, Debaryomyces hansenii, Ogataea parapolymorpha, and Dekkera bruxellensis, were found associated with the starter culture along with a diverse range of opportunistic contaminants. The bacterial microflora was dominated by lactic acid bacteria (LAB). The most frequent occurring LAB was Lactobacillus plantarum, Lactobacillus brevis, Leuconostoc lactis, Weissella cibaria, Lactococcus lactis, Weissella para mesenteroides, Leuconostoc pseudomesenteroides, etc. Our study provided a comprehensive picture of microbial diversity associated with rice wine fermentation starter and indicated the superiority of metagenomic sequencing over previously used techniques.
\end{abstract}

Madhumita Barooah

m17barooah@yahoo.co.in

1 Department of Agricultural Biotechnology, Assam Agricultural University, Jorhat 785013, Assam, India
Keywords Metagenomics - Starter culture - Taxonomy · Rice wine and microflora

\section{Introduction}

The methodology of rice wine preparation is more or less similar among most of the ethnic communities of Assam, a north-eastern state of India; however, fermentation starters (Assamese name xaj-pitha) and the substrates differ resulting in variance in the quality of the final product (Tanti et al. 2010; Das et al. 2012). The use of fermentation starters is believed to have originated in China from where it spread to the other neighboring countries in Asia (Hanai 1992). Various local names for rice wine starters are used in Asian countries, such as banh men in Vietnam, chu in China, koji in Japan, nuruk in Korea, murcha in India, ragi in Indonesia, ragi tapai in Malaysia, and bubod in Philippines (Limtong et al. 2005). In Assam, different ethnic communities also have their own terms to refer to such fermentation starter cultures. The starters are mixed cultures of molds, yeasts, and bacteria that are maintained on substrate, such as rice powder, supplemented with various herbs.

The indigenous microbial diversity in various sources of local beverages could be a rich resource for oenological research. Few studies have reported microbial diversity of rice wine fermentation starters (Mao and Odyuo 2007; Xie et al. 2007; Rong et al. 2009; Shi et al. 2009). However, most of these studies examined the microbial community of wine starters using culture-dependent methods that might not have permitted identification of some important microbial species, as their growth requirements are unknown, and hence, a comprehensive picture of the microbial diversity in rice wine starters is yet to be reported (Thanh et al. 2008). 
The first culture-independent study of starter microbiota was reported in the traditional Vietnamese alcohol fermentation starters (banh men) through Polymerase Chain Reaction (PCR)-based Denaturing Gradient Gel Electrophoresis (DGGE) (Thanh et al. 2008). The study revealed a remarkable diversity of fungal microflora among various samples; however, the bacterial community exhibited a rather "spontaneous" species composition among the collected starters. Although cultureindependent approaches, such as Polymerase Chain Reaction (PCR)-based amplification and sequencing of 16S rRNA genes or Denaturing Gradient Gel Electrophoresis (DGGE), have proven to be powerful tools in investigating different types of traditional fermentations (Meroth et al. 2003; Prakitchaiwattana et al. 2004; Rantsiou et al. 2005; Haruta et al. 2006), the technique is unable to reflect the overall microbial diversity due to low throughput (Ercolini 2004; Prakitchaiwattana et al. 2004). On the other hand, high-throughput sequencing (HTS) methods, such as 454 pyrosequencing and Illumina sequencing technologies, have been recently applied as novel promising methods to investigate microbial communities in different habitats. DNA-based high-throughput-sequencing metagenomics have been applied to reveal microbial communities in marine water (Gilbert et al. 2008), soil (Urich et al. 2008), oral cavities (Lazarevic et al. 2009), human guts (Qin et al. 2010), etc. HTS has also been used for exploring the microbial community structure of a variety of fermented foods and beverages; e.g., Kimchi, a traditional Korean fermented food (Jung et al. 2011), Irish soft, semi-hard, and hard cheeses (Quigley et al. 2012), Danish raw milk cheeses (Masoud et al. 2011), Kefir grains (used in fermentation of Kefir, a traditional Turkish drink) (Nalbantoglu et al. 2014), Chinese rice wine (Hong et al. 2016), etc. Moreover, HTS was also instrumental in unveiling the microbial succession of Lactobacillales and yeasts over the members of the Enterobacteriaceae in brewing American coolship ale (Bokulich et al. 2012). Based on the HTS analysis and carbohydrate utilization pattern, Lactobacillus fabifermentans was identified to be one of the most dominant bacterial species involved in grape marc fermentation (Campanaro et al. 2014). Based on these observations, it can be concluded that the HTS analysis could provide more insights into the microbial communities and also reflects their role on fermentation processes.

However, studies on microbial community diversity through metagenomics approach in rice wine culture is yet to be reported. Our study is the first of its kind to use the whole genome shotgun (WGS) sequencing to analyze the microbial community occurring in the starter culture traditionally used in Assamese rice wine fermentation. We aimed at identifying the key microbial communities associated with traditional starter culture (xaj-pitha) and attempted to find relatedness of the microbiota with that of the previously analyzed starters from south-east Asia.

\section{Materials and methods}

The starter is prepared by concoction of rice flour and several herbs. These herbs are believed to impart intoxicating property to the liquor (Sarma 2002). Apart from contributing various organoleptic properties to the wine, these various plants are also said to have many other medicinal properties (Das et al. 2012). Some of the plant extracts may also provide certain nutrients for the survival and growth of the microflora present in the starter cakes (Thanh et al. 2008).

The fermentation in rice wine is known to be a consortia effect of several biochemical and ecological processes, where yeast strains play a major role in ultimate conversion of fermentable sugar to alcohol and esters. Usually, glutinous rice (local name Bora) is first steamed and then allowed to cool on a bamboo mat. Powdered starter (four starter cultures per $\mathrm{kg}$ of rice) is sprinkled on the cooked rice and mixed thoroughly. This mixture is packed into an earthen pot, properly sealed with fresh leaves of Dryopteris sp. (local name dhekia). Several of these pots are kept atop of a top bench (local name dhowa-chang) over fire place. Incubation results in the production of a light-yellow alcoholic beverage, called as rohi, are collected from the pot. Rohi has a sweet taste and a strong aroma. It is decanted and diluted with water to serve as $x a j$.

\section{Sampling}

Five rice wine starter samples belonging to rural households were collected from the Titabar sub-division $\left(26.60^{\circ} \mathrm{N}, 94.20^{\circ} \mathrm{E}\right.$ ) of Jorhat district (state of Assam, India) on August 20, 2014. All the samples were collected from people belonging to Ahom community of the study area. The Ahoms of the Tai-Shan family came from Burma across the Patkai range and entered Assam under an adventurous leader Sukapha. The special section to which they belonged, or the Shans proper, occupied the northern and eastern hill tracts of Upper Burma and Western Yunnan, where they formed a group of states for which, according to Ney Elias, there is no collective native name. The Ahoms subdued the various local chiefs through a series of determined and skilful moves, and very soon firmly entrenched themselves as the masters over a long tract. Skilled women from rural background of this community are engaged in the traditional $\mathrm{Xaj}$ preparation. 
Table 1 Biochemical composition of starter cultures collected from Titabar sub-division, Jorhat district, Assam, India

\begin{tabular}{lllllllllll}
\hline $\begin{array}{l}\text { Sample } \\
\text { code }\end{array}$ & $\begin{array}{l}\text { Weight in } \\
\text { gm/shape }\end{array}$ & Moisture & $\begin{array}{l}\text { Crude } \\
\text { fat } \%\end{array}$ & $\begin{array}{l}\text { Crude } \\
\text { protein } \%\end{array}$ & $\begin{array}{l}\text { Crude } \\
\text { fiber } \%\end{array}$ & Ash $\%$ & Starch \% & $\begin{array}{l}\text { Total soluble } \\
\text { sugar } \%\end{array}$ & $\begin{array}{l}\text { Reducing } \\
\text { sugar } \%\end{array}$ & $\begin{array}{l}\text { Non-reducing } \\
\text { sugar } \%\end{array}$ \\
\hline ABT-S4J3 & $11.598 /$ round & 13.61 & 0.76 & 7.48 & 1.863 & 1.13 & 74.58 & 1.211 & 0.297 & 0.914 \\
ABT-S5J3 & 12.024 oval & 14.46 & 0.96 & 8.74 & 2.006 & 1.27 & 72.39 & 1.267 & 0.276 & 0.991 \\
ABT-S6J3 & $12.560 /$ oval & 14.34 & 0.87 & 8.19 & 1.762 & 1.14 & 73.37 & 1.013 & 0.345 & 0.668 \\
ABT-S7J3 & $14.462 /$ round & 13.89 & 0.86 & 7.36 & 1.634 & 0.83 & 75.29 & 1.027 & 0.297 & 0.730 \\
ABT-S8J3 & $12.220 /$ oval & 13.21 & 0.76 & 6.38 & 2.224 & 0.61 & 76.78 & 1.164 & 0.067 & 1.097 \\
\hline
\end{tabular}

The collected samples were transported to the laboratory on ice packs and were immediately subjected for the proximate analysis using the standard procedure. Moisture, crude fat, and ash contents of collected starter samples were determined through the standard methods (AOAC 1970). The total nitrogen content of fat-free samples was estimated by the Micro-Kjeldahl method, and crude protein was calculated by multiplying the total $N$ by 6.25 (Balasubramaniam and Sadasivam 1987). Crude fiber was determined using the method described by Sadasivam and Manickam (1996). Total soluble sugar was determined using the Anthrone method (Yemm and Willis 1954). The reducing sugar was estimated by the standard biochemical method using 3,5-di-nitrosalicylic acid (DNS) reagent with slight modifications (Somogyi 1952). The non-reducing sugar content was derived by subtracting the percentage of reducing sugar from the percentage of total soluble sugar. Results of biochemical composition of the samples have been presented in Table 1. One gram of starter was weighed from each powdered sample and then pooled as a composite sample in sterile laboratory conditions.

\section{DNA isolation from starter samples}

DNA was isolated from $1 \mathrm{~g}$ of composite starter sample using Environmental gDNA isolation kit (Xcelgen, India). Extracted DNA was quantified using Qubit fluorometer according to manufacturer's instructions.

\section{Preparation of $2 \times 300$ MiSeq library}

The paired-end sequencing library was prepared using illumina TruSeq DNA Library Preparation Kit, initiated with fragmentation of $1 \mu \mathrm{g}$ gDNA, followed by paired-end adapter ligation. The ligated product was purified using $1 \mathrm{X}$ Ampure beads. The purified product was subjected to sizeselection at $\sim 500-800 \mathrm{bp}$, and the selected product was PCR amplified as described in the kit protocol. The amplified library was analyzed in Bioanalyzer 2100 (Agilent Technologies) using high-sensitivity (HS) DNA chip as per manufacturer's instructions.

\section{Cluster generation and sequencing}

After obtaining the Qubit concentration for the library and the mean peak size from Bioanalyzer profile, $10 \mathrm{pM}$ of library was loaded onto Illumina MiSeq for cluster generation and sequencing. Paired-end sequencing allows the template fragments to be sequenced in both the forward and reverse directions on MiSeq. The reagents supplied with the kit were used in the binding of samples to complementary adapter oligos on paired-on flow cell. The adapters were designed to allow selective cleavage of the forward strands after resynthesis of the reverse strand during sequencing. The copied reverse strand was then used to sequence from the opposite end of the fragment. High-quality metagenome reads were assembled using CLC workbench (CLC bio, Denmark) at default parameter (minimum contig length 200) for trimming and de novo assembly (Chan et al. 2012). In Fig. 1, we summarize our analysis strategy.

\section{Domain information analysis}

Taxonomic domain information analysis was conducted at the MG-RAST (Meta Genome Rapid Annotation using Subsystem Technology, v3.1) server at the Argonne National Library (http://metagenomics.nmpdr.org). Species richness was computed as the antilog of the Shannon diversity (Meyer et al. 2008). A single similarity search at this server allows retrieving similarities to several databases, including NCBI-nr, KEGG, SEED, egg-NOG, COG, etc.

\section{Taxonomic classification}

Taxonomic classification was conducted by BLASTN against SILVA SSUref and LSUref databases release 108 with an $e$ value of $1 \mathrm{e}^{-5}$ (Urich et al. 2008) followed by the annotation of BLAST output files using MEGAN (Huson et al. 2007). This was performed by the lowest common ancestor algorithm that assigned rDNA or rRNA sequences to the lowest common ancestor in the taxonomy from a subset of the best scoring matches in the BLAST result 


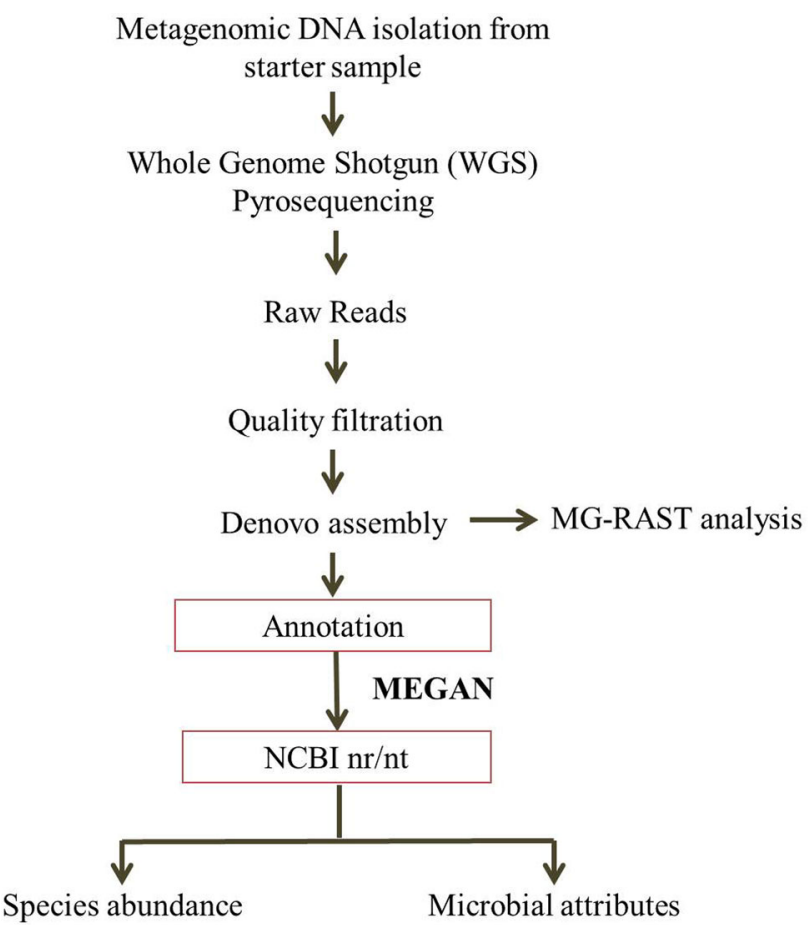

Fig. 1 Analysis strategy performed to analyze microbial diversity prevalent in the starter culture sample. DNA from an efficient starter sample was used for whole genome shotgun (WGS) sequencing

(absolute cutoff BLAST bitscore 86, relative cutoff $10 \%$ of the top hit) (Urich et al. 2008) using MEGAN according to these cutoffs to select hit reads for annotation. Randomly sequence reads exhibit very different levels of evolutionary conservation; therefore, it is important to make use of all ranks of the NCBI taxonomy, placing more conserved sequences higher up in the taxonomy (i.e., closer to the root) and more distinct sequences onto nodes that are more specific (i.e., closer to the leaves, which represent species and strains)

\section{Results}

\section{Sequence analysis}

Whole genome shotgun sequencing of the starter sample revealed 278,231 sequences containing 143,119,277 base pairs (bp) rendering an average read length of $641 \mathrm{bp}$. A total of 5302 sequences $(1.9 \%)$ failed to pass the qualitycontrol (QC) pipeline. Of the sequences that passed QC, 590 sequences $(0.2 \%)$ contained ribosomal RNA genes. The remaining 119,194 sequences $(42.8 \%)$ contained predicted proteins with known functions and 132,223 sequences $(47.5 \%)$ contained predicted proteins with unknown function. About 20,917 (7.5\%) of the sequences that passed QC had no rRNA genes or predicted proteins.
Out of the 272,929 sequences (totaling 143,119,277 bps) that passed quality control, 251,417 $(92.1 \%)$ produced 305,993 predicted protein coding regions. Of the 305,993 predicted protein features, a total of 114,064 (37.3\%) sequence reads had annotation with at least one of the protein databases (M5NR) and 191,929 (62.7\% of features) had no significant similarities to any protein databases, and about 61,377 features $(53.8 \%$ of annotated features) were assigned with functional categories, as described in the flow chart (Fig. 2; Table 2).

\section{Metagenome phylotyping (MG-RAST ID: 4556318.3)}

Cluster of Orthologous Groups (COGs) had 52,245 sequences predicted functions. Out of which, $21 \%$ were identified with cellular processes and signaling, $21.7 \%$ performed to information storage and processing, and $34.9 \%$ could be attributed to metabolism functional categories. KEGG Orthology (KO) Functional Category had 31,626 sequences with predicted functions. Out of which, $46.9 \%$ could be assigned to metabolism, $22.7 \%$ to genetic information processing, $14.3 \%$ to environmental information processing, and $11.8 \%$ to cellular processes. Subsystem Functional Category had 47,970 sequences with predictable functions. $10.2 \%$ of these sequences were

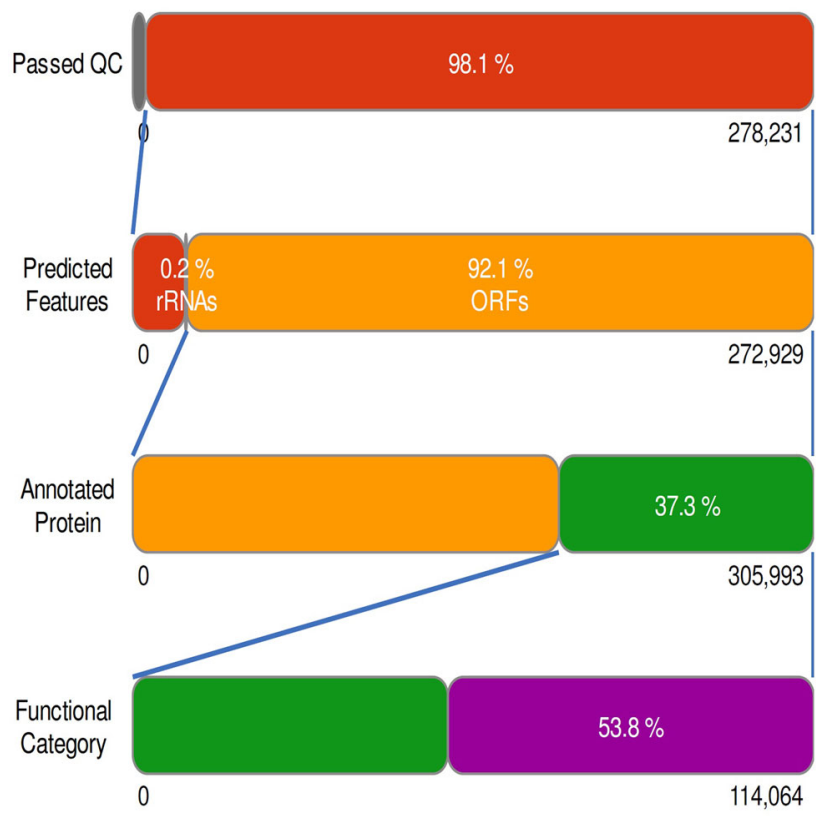

Fig. 2 Flow chart depicting functional category hit distribution. 5302 sequences failed quality control. Of those, de-replication identified 1240 sequences $(0.4 \%$ of total) as artificial duplicate reads (ADRs). These include protein databases, protein databases with functional hierarchy information, and ribosomal RNA databases. The bars representing annotated reads are colored by $e$ value range 
Table 2 Sequence statistics for the starter sample covering raw and high-quality sequence data used in the downstream analysis

\begin{tabular}{ll}
\hline Analysis statistics & Values (in number) \\
\hline Number of contig & 278,231 \\
Total contig length & $178,534,809$ \\
Maximum contig length & 213,370 \\
Minimum contig length & 275 \\
Base-pair count & $178,540,333$ \\
Mean sequence length & $641 \pm 1650$ \\
Mean GC percent & $42 \pm 10$ \\
Artificial Duplicate Reads (ADRs) & 1240 \\
Post QC base-pair count & $143,119,277$ \\
Post QC sequence count & 272,929 \\
Mean sequence length & $524 \pm 397$ \\
Predicted protein features & 305,993 \\
Predicted rRNA features & 35,780 \\
Identified protein features & 114,064 \\
Identified rRNA features & 476 \\
Identified functional categories & 61,377 \\
\hline
\end{tabular}

associated with carbohydrates, $8.1 \%$ with protein metabolism, and $7.5 \%$ with amino acid derivatives functional categories (Fig. 3). Of the sequences that passed QC, 590 sequences $(0.2 \%)$ contained ribosomal RNA genes. MGRAST analysis showed that at domain level, Eukaryota were the dominant domain, accounting for $60.4 \%$ sequences, while bacteria comprised approximately $39.2 \%$. Sequences from viruses and others only accounted for $0.4 \%$. The genus-level assignments made by MGRAST are shown in Fig. 3a. Among microbes, the genus Lactobacillus dominated the profile with $7.23 \%$ total abundance, followed by the genre Pseudomoonas (6.59\%), Acidovorax (4.17\%), Saccharomyces (3.80\%), and Leuconostoc $(2.98 \%)$ with varying but comparable abundance.

At phylum level, a total of $38.7 \%$ sequences belonged to streptophyta, $23 \%$ sequences belonged to proteobacteria, and $15.8 \%$ belonged to ascomycota. Taxonomic distribution at family level revealed that a total of $32.4 \%$ sequences belonged to poaceae, $7.7 \%$ belonged to lactobacillaceae, and $6.7 \%$ sequences belonged to pseudomonadaceae (Fig. 4).

The assembled contigs were subjected to annotation using the LCA algorithm. The annotation was performed by aligning the contigs to non-redundant database of NCBI using BLASTX. These annotated files were imported to MEGAN v.5.2.3, and the downstream analysis was carried out. In Fig. 5, we show the LCA plot of these species based on the SSU rRNA analysis. Maximum contigs were assigned to Oryza sativa (26,856 contigs), followed by Rhizopus delemar (6660 contigs), Mucor circinelloides (6082 contigs), Lactobacillus plantarum (2194 contigs), Meyerozyma guilliermondii (1693 contigs), Stenotrophomonas maltophilia (1644 contigs), Wickerhamomyces ciferrii (1304 contigs), and Lactobacillus brevis (1054 contigs).

\section{Discussion}

Rice wine is popular among most of the ethnic communities of Assam. They are prepared and consumed during various religious and harvest festivals. The preparation of rice wine involves the conversion of cooked rice by physical, microbiological, and biochemical operations, including steaming, inoculation with starter, and fermentation. A wide range of microorganisms are involved during fermentation processes, but only a few determines the quality of the endproduct. Three major microbial groups, namely molds, yeasts, and lactic acid bacteria, are reported to be involved in the traditional rice wine starters (Hesseltine et al. 1988; Steinkraus 1989; Lim 1991). Rice wine fermentation basically involves two major steps, viz., liquefaction and saccharification and alcoholic fermentation. In the first step, i.e., liquefaction and saccharification, fermentable sugars are produced from starch by the action of a-amylase and amyloglucosidase from molds which occurs as aerobic solid-state fermentation proceeds. Some yeast can also degrade starch, but this trait is not widespread (Laluce and Mattoon 1984; De Mot et al. 1985). The endproducts in this step are mainly glucose, and also to some extent dextrins and maltose (Crabb 1999). The second step of rice wine fermentation, i.e., alcoholic fermentation, involves conversion of fermentable sugars into alcohol by various yeasts. Some molds are also efficient in alcoholic fermentation. Different bacteria, mainly lactic acid bacteria (LAB), occur as opportunistic contaminants (Gandjar 1999; Thanh et al. 2008).

Controlled rice wine fermentation can be carried out using commercial or defined mixed starters which contain well-characterized efficient fungal and yeast strains (Dung et al. 2005). However, such products lose their traditional, characteristic taste, and odor. An ideal starter culture should enable obtaining fermented products with the palatability equal to good quality products, obtained as a result of spontaneous fermentation (Jazwiak et al. 2013).

\section{Microbial diversity and abundance}

MG-RAST analysis of the metagenomic sequences revealed an alpha-diversity of 68.836 species. The rarefaction curve, which shows the observed operational taxonomic unit (OTU) richness as a function of the number of sequences sampled, indicated that the sequencing depth was insufficient to wholly capture the diversity present, indicating that the current sampling of the communities is 


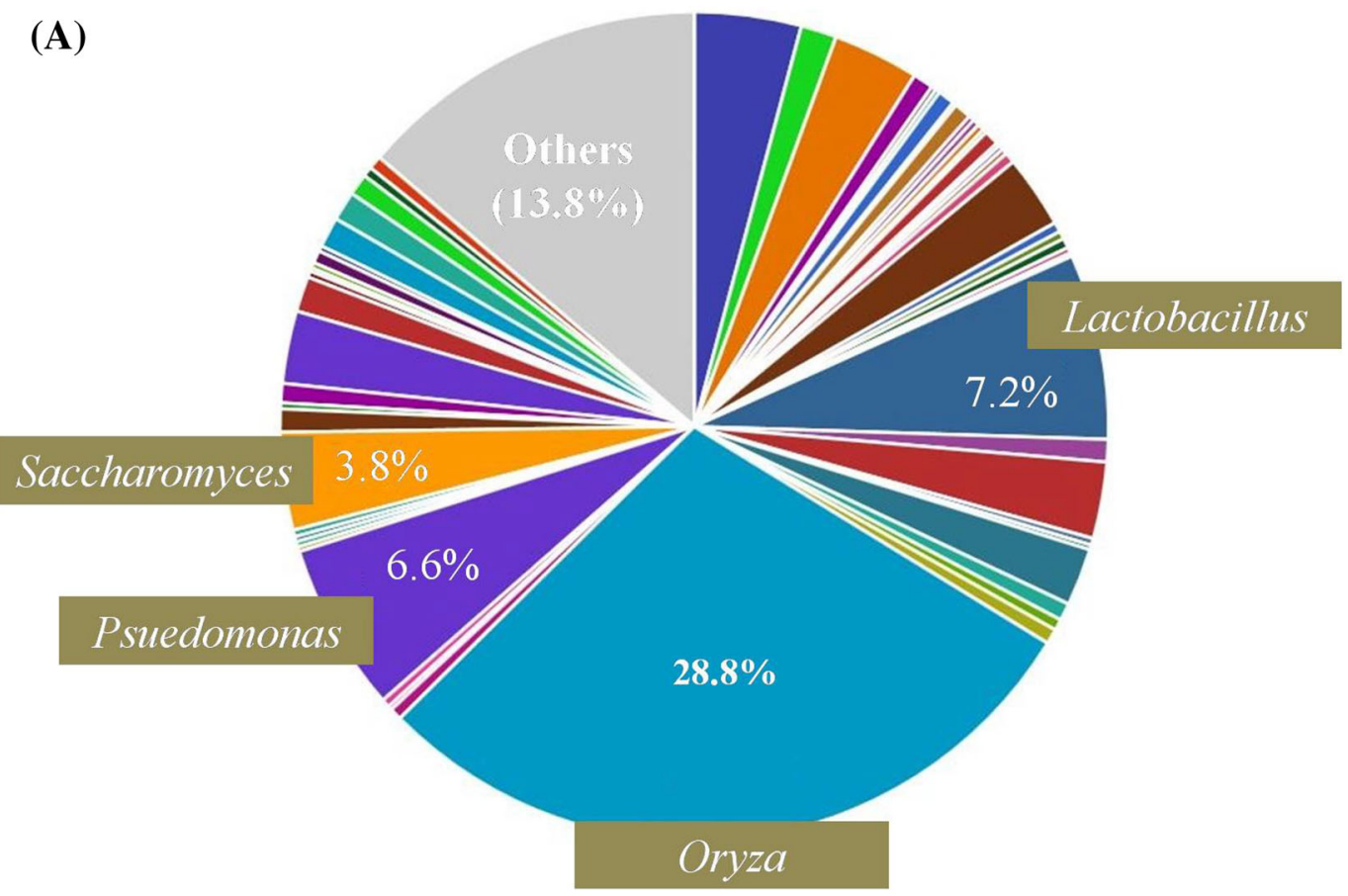

(B)

Virulence, Disease and Defense

Sulfur Metabolism

Stress Response Secondary Metabolism Respiration Regulation and Cell signaling RNA Metabolism

Protein Metabolism Potassium metabolism

Photosynthesis

Phosphorus Metabolism

Phages, Prophages, Transposable elements, Plasmids Nucleosides and Nucleotides

Nitrogen Metabolism

Motility and Chemotaxis Miscellaneous

Metabolism of Aromatic Compounds Membrane Transport Iron acquisition and metabolism Fatty Acids, Lipids, and Isoprenoids Dormancy and Sporulation DNA Metabolism Cofactors, Vitamins, Prosthetic Groups, Pigments Clustering-based subsystems Cell Wall and Capsule Cell Division and Cell Cycle Carbohydrates Amino Acids and Derivatives

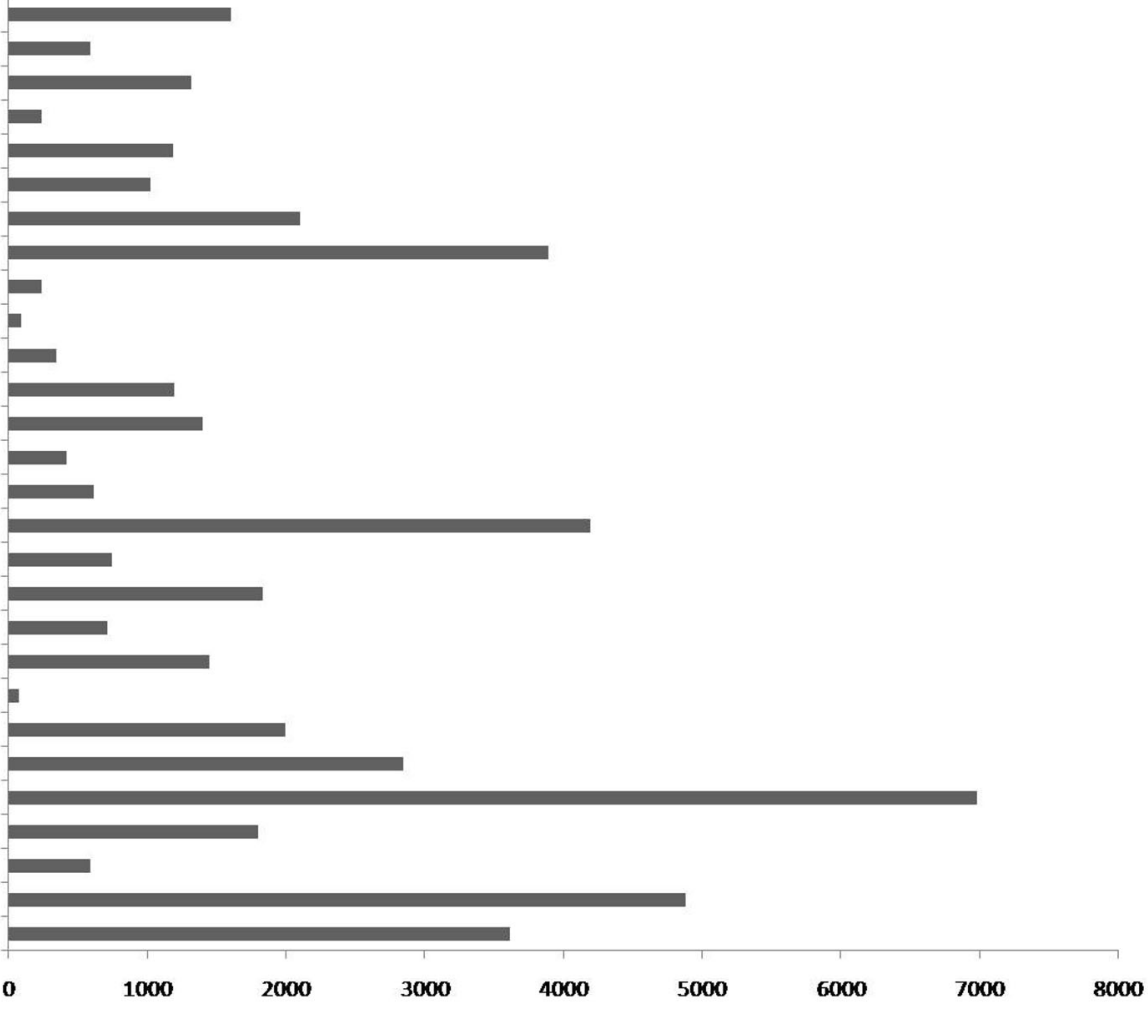

Abundance in the metagenome (Hit)

Fig. 3 MG-RAST analyses of starter sample WGS metagenomics sequences. a Genus-level assignment of the sequences; b Functional annotation of predicted protein sequences 


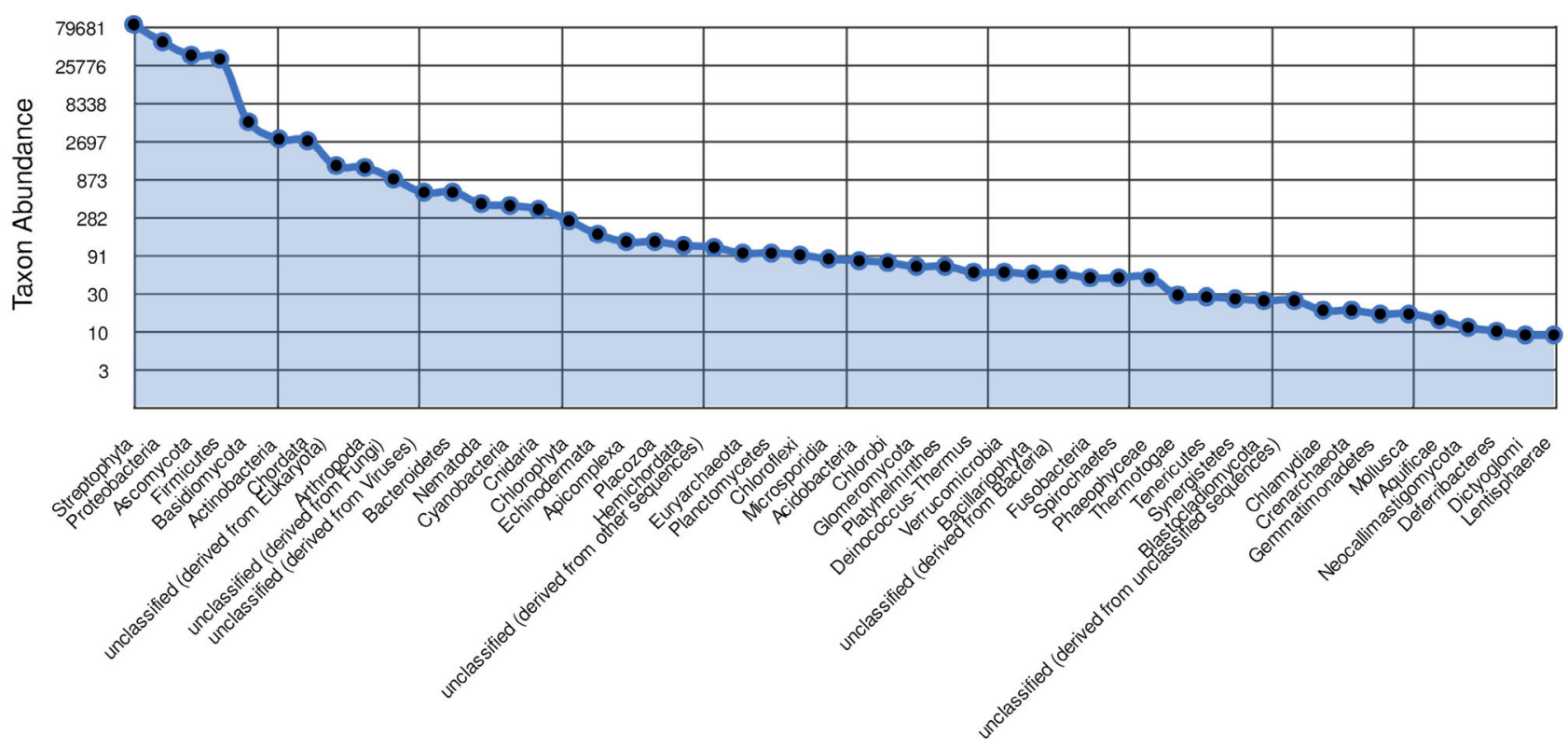

Fig. 4 Phylum abundance ordered from the most abundant to least abundant. Only the top 50 most abundant are shown. The $Y$-axis plots the abundances of annotations in each phylum on a log scale. The

insufficient and a large fraction of the species diversity remains to be discovered with more intensive sampling.

\section{Fungal diversity prevalent in xaj-pitha}

Amylolytic fungi, such as Mucor sp., Rhizopus sp., Amylomyces rouxii, etc., have already been reported to be dominant in bakhar, a reported Indian starter culture (Tamang and Sarkar 1995). PCR-DGGE analysis revealed that four species of filamentous fungi belonging to the family Mucoraceae (Rhizopus oryzae, Rhizopus microsporus, Absidia corymbifera, and Amylomyces sp.) were dominant in traditional Vietnamese starter culture, "banh men" (Thanh et al. 2008). All these filamentous fungi are strong amylase producers and are frequently found in Asian fermentation starters (Hesseltine 1983; Hesseltine et al. 1988; Haard et al. 1999). Dominant fungal diversity that occurred in "hong qu starter" (starter for Chinese glutinous rice wine) is $R$. oryzae, $R$. microspores, Mucor circinelloides, Penicillium chrysogenum, Aspergillus awamori, Aspergillus niger, Aspergillus flavus, Aspergillus oryzae, Monascus purpureus, and Monascus ruber (Lv et al. 2013).

Three species of amylolytic filamentous fungi viz., Rhizopus delemar, Mucor circinelloides (Family: Mucoraceae), and Aspergillus sp. (Family: Trichocomaceae), were found in the present study of "xaj-pitha". Mucor mycotina, including Rhizopus, Amylomyces, and Mucor, are indispensible microorganisms for the production of fermented foods in East Asia. Members of the genus Rhizopus specifically have often been reported from various rank abundance curve is a tool for visually representing taxonomic richness and evenness

fermented foods in eastern and southeastern Asia. Taxonomic contribution from Schipper and Stalpers (1984), Schipper (1984), Yuan and Jong (1984), Ellis (1985), Weitzman et al. (1996) etc., has consolidated the presence of 13 species in the group. $R$. oryzae is the most common fungus of any kind in the community prevalent in banh men, having been identified in 47 out of 52 tested samples. Rhizopus species, such as $R$. oryzae strains $28,168,28,627$, and 34,612, exhibited $\alpha$-amylase activity (42.2, 76.0, and $40.4 \mathrm{U} \mathrm{g}^{-1}$, respectively) in solid-state fermentation (Soccol et al. 1994). During fermentation, this fungus binds dehulled and cooked substrate into a solid cake covered with a dense cottony mycelium.

Mucor circinelloides produces a complete set of cellulose degrading enzymes (Huang et al. 2014), indicating that this fungus could potentially be used in biomass conversion. However, Mucor circinelloides has also been identified as the causal agent of primary invasive cutaneous and maxillofacial zygomycosis (Iwen et al. 2007; Khan et al. 2009). Therefore, safety issues related to the presence of Mucor circinelloides in ethnic starters should be examined carefully.

\section{Yeast diversity prevalent in $x a j$-pitha}

In a wine making industry, yeasts play the most significant role, as they are the key to alcoholic fermentation. Major yeast diversity found in xaj-pitha were Meyerozyma guilliermondii, Wickerhamomyces ciferrii, Saccharomyces cerevisiae, Candida glabrata, Debaryomyces hansenii, 


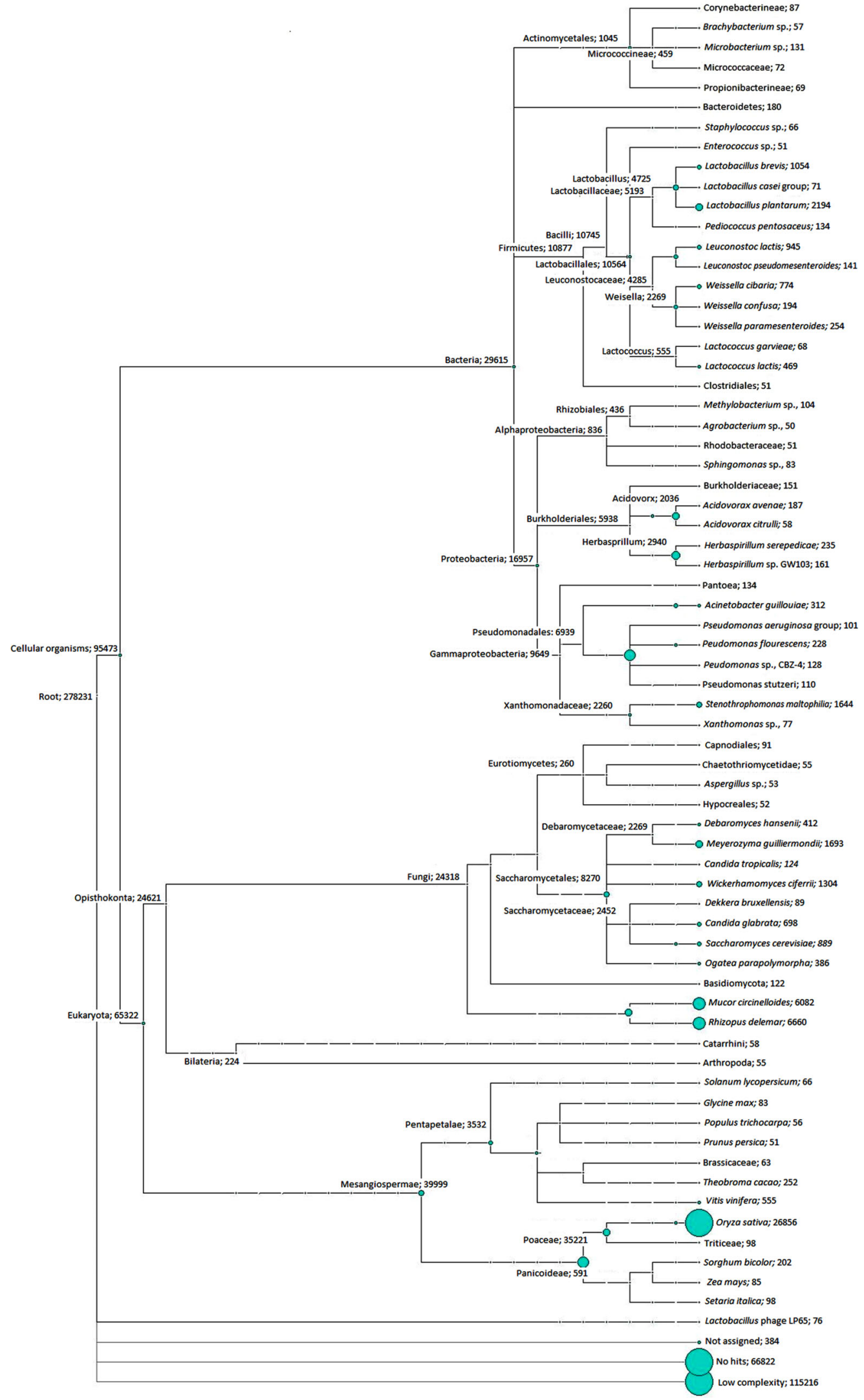


4Fig. 5 Phylogenetic diversity was computed using the LCA algorithm based on a BLASTX comparison of all the contigs against the NCBI-NR database. Each circle represents a taxon in the NCBI taxonomy and is labeled by its name and the number of contigs that are assigned either directly to the taxon, or indirectly via one of its subtaxa. The size of the circle is scaled logarithmically to represent the number of contigs assigned directly to the taxon

Ogataea parapolymorpha, and Dekkera bruxellensis (in descending order of their relative abundance). Previous studies have reported that Saccharomycopsis fibuligera, $S$. cerevisiae, Wickerhamomyces anomala, Pichia guilliermondii, and Candida sp. are the most common yeasts in rice wine starters (Lee and Fujio 1999; Xie et al. 2007; Jeyarama et al. 2008). A comprehensive assessment of yeast diversity occurred with "hong qu starter" (starter for Chinese glutinous rice wine) revealed the presence of Saccharomyces cerevisiae, Saccharomycopsis fibuligera, Pichia fabianii, Candida glabrata, Cryptococcus heveanensis, Cryptococcus albidus, Saccharomycopsis malanga, Rhodotorula mucilaginosa, Sporobolomyces nylandii, Wickerhamomyces anomalus, and Rhodosporidium toruloides (Lv et al. 2013). On the other hand, Saccharomycopsis fibuligera, Saccharomyces cerevisiae, Issatchenkia sp., Pichia anomala, Clavispora lusitaniae, Candida tropicalis, and Pichia ranongensis were the yeast species found to occur in traditional Vietnamese alcohol fermentation starter "banh men" (Thanh et al. 2008). We assume that higher yeast diversity as reported in these studies could have resulted from a larger sampling population.

The yeast, Saccharomycopsis (Endomycopsis) fibuligera, possessing amylolytic and ethanol producing capacity, is one of the common yeasts present in traditional rice wine starter (Limtong et al. 2002). In our study, Saccharomycopsis fibuligera could not be detected in the starter. However, the predominant yeasts in alcoholic fermentation belong to the genus Saccharomyces, especially S. cerevisiae (Battcock and Ali 1993). It is the most effective ethanol producer known so far (Vaughan-Martini and Martini 1995). Meyerozyma guilliermondii (previously named Pichia guilliermondii) has been found to be effective towards different postharvest spoilage fungi, such as Penicillium digitatum on grapefruit (Droby et al. 1989), Botrytis cinerea and Penicillium expansum on apples (Wisniewski et al. 1991), and Aspergillus flavus on soybeans (Paster et al. 1993). The yeast may act as a biocontrol agent against spoilage microorganisms increasing the shelf life of the product. The antagonistic effect shown by the yeast against $P$. digitatum and $B$. cinerea is mainly due to the competition for nutrients and the secretion of cell wall degrading enzymes (Petersson and Schnürer 1995).

Debaryomyces hansenii is extremophilic yeast; it has been isolated from food products, such as cheese, meat, wine, beer, fruit, etc. (Norkrans 1966; Davenport 1980;
Barnett et al. 2000) as well as from some other high-sugar products (Tilbury 1980). This high lipid accumulating, osmotolerant (Onishi 1963) yeast has shown some interesting genetical and biochemical features for upcoming biotechnological applications (Baronian 2004; Ratledge and Tan 1990). Unfortunately, D. hansenii is also well known for spoilage of brine-preserved foods, such as gherkins (Breuer and Harms 2006).

A wide variety of spoilage yeasts, e.g., Pichia sp., Zygosaccharomyces sp., Kluyveromyces sp., Brettanomyces sp., etc., have also been reported from alcoholic fermentation, which may spoil wines during storage through changes of biochemical activities (Fleet 1993). Out of these, members of the genus Brettanomyces (imperfect state, Dekkera) are probably the most serious and controversial spoilage yeasts (Cocolin et al. 2004). These yeasts have been reported from almost every wine-producing area of the world (Fungelsang 1997). They have also been isolated from other fermented beverages, such as beer and cider. Osmo-/alcohol-tolerant Brettanomyces species can survive and contaminate wines in an ill-managed setup. Brettanomyces/Dekkera-contaminated wine develops offflavors and a distinct haziness, which severely decreases product market value (Chatonnet et al. 1992, 1995; Edlin et al. 1995).

\section{Lactic acid bacteria (LAB) prevalent in xaj-pitha}

The group of Lactic acid bacteria (LAB) was found to be the most diverse bacterial as well microbial group with 4 genera and 12 species detected. The following species were found to be most abundant: Lactobacillus plantarum, Lactobacillus brevis, Leuconostoc lactis, Weissella cibaria, Lactococcus lactis, Weissella para mesenteroides, Weissella confusa, Leuconostoc pseudomesenteroides, Pediococcus pentosus, Lactobacillus casei group, Lactococcus garvieae, and Enterococcus sp. Major LAB diversity viz, Weissella paramesenteroides, Pediococcus pentosaceus, and Pediococcus acidilactici have been reported to occur in "hong qu starter" (a Chinese glutinous rice wine) (Lv et al. 2013). On the other hand, Enterococcus faecium, Lactobacillus agilis, Lactobacillus brevis, Lactobacillus fermentum, Lactobacillus manihotivorans, Lactobacillus plantarum, Lactobacillus sp., Lactococcus lactis, Leuconostoc citreum, Leuconostoc garlicum, Leuconostoc mesenteroides, Pediococcus pentosaceus, Weissella confusa, and Weissella paramesenteroides have been reported from traditional Vietnamese alcohol fermentation starter Banh men (Thanh et al. 2008).

Acidification caused by this group of bacteria favors the growth of amylolytic yeast and fungi while suppressing the growth of unwanted spoilage and pathogenic bacteria (Gandjar 1999; Haard et al. 1999). Probably abundance of 
$\mathrm{LAB}$ is also the reason behind most of the off-flavored and hazy colored rice wine with low shelf life. Many of these Lactobacillus strains are already in the pharmaceutical industry as probiotics agents; however, their credibility as good probiotics for human usage is still under debate and needs further clinically supporting data (Reid 1999).

\section{Plant pathogens/environmental contaminants}

The major plant pathogens viz., Acidovorax avenae, Acidovorax avenae subsp. avenae (Pseudomonas avenae), Acidovorax avenae subsp. citrulli, Herbaspirillum seropedicae, Herbaspirillum sp. GW103, Pantoea, Methylobacterium, Sphingomonas, Xanthomonas, etc., were detected in the sample. Some other environmental (from soil and water) contaminants, such as Pseudomonas fluorescens, Pseudomonas sp. CBZ-4, Pseudomonas stutzeri, Psuedomonas aeruginosa group, Stenotrophomonas maltophil$i a$, etc., were also detected. Ethnic fermentation process is carried out at rural household level, where hygiene is often compromised. Several opportunistic human skin commensals, such as Acinetobacter guillouiae, Microbacterium sp., Micrococcus sp., Staphylococcus sp., etc., were detected. Out of these, Acinetobacter guillouiae has received increasing attention during the recent times as significant opportunistic pathogen, usually in the context of serious underlying disease in immune-compromised patients from south-east Asia and tropical Australia (Dijkshoorn et al. 2007, Peleg et al. 2008). We presume that all contaminants (plant pathogen/environmental contaminant/clinical contaminant) in the starter were introduced along with the plant parts, or through unhygienic practices.

\section{Herbs}

It has been observed that different rice wine-producing communities follow almost identical protocols for fermentation, but they use different species of plants in the starter culture preparation, which are believed to add as an intoxicating property to the liquor (Sarma 2002). Ethnic communities of North East India have been regularly using several herbs and plants, such as Albizia myriophylla, Amomum aromaticum, Plumbago zeylanica, Buddleja asiatica, Vernonia cinerea, Gingiber officinale, Glycyrrhiza glabra, Ananas comosus, Artocarpus heterophyllus, Calotropis gigantea, Capsicum frutescens, etc. (Das et al. 2012). In South Asian country Cambodia, spice plants, such as Capsicum sp., Alpinia sp., Piper sp., Allium sativum, Zingiber officinale, Illicium verum, Amomum krervanh, Cinnamomum sp., etc., and sweet ingredients (Albizia sp., Cinnamomum sp., Saccharum officinarum) are commonly used (Das et al. 2012; Seesuriyachan 2011). Apart from imparting color, flavor, and sweetness to the wine, the various plants used in the starter culture are also said to have many medicinal properties. Some of the plant extracts may also provide certain nutrients for the survival of the microflora present in the starter cakes. The effects of the herbs used in traditional starter preparations on the starter microflora have been studied (Phuc 1998; Dung et al. 2005). It was suggested that some herbs have a stimulatory effect on biomass and also on yeasts count. Particularly, the herbs "Tieu Hoi" (Fennel: Foeniculum vulgare Miller) and "Dinh Huong" (Clove: Syzygium aromaticum L.) used in Vietnamese starter culture prove to be stimulatory in biomass production of molds and yeasts (Dung et al. 2005). Our metagenomics study has revealed the presence of several plant parts viz., Prunus persica, Setaria italica, Glycine max, Solanum lycopersicum, Brassica sp., Vitis vinifera, etc., in the starter. However, any stimulatory or inhibitory effects of these plant parts are yet to be tested. Nonetheless, Ppdfn1, a defensin protein, from Prunus persica (common name peach) shows antifungal activity against pathogens, such as Botrytis cinerea, Monilinia laxa, and Penicillium expansum (Nanni et al. 2013). Interestingly, phyllosphere of Setaria italica has been reported to harbor some of the dominant fungi, such as Rhizopus nigricans, Curvularia pallescens, Aspergillus flavus, A. fumigatus, and Trichoderma album (Upadhyaya and Gupta 2009). This indicates that the herbs and other plants may also be a source of some of the microbial mass which could play an important role in fermentation. At this point, we assume that a study on microbial dynamics, including succession and niche differentiation, during the actual alcohol fermentation could be an interesting topic for further research.

\section{Conclusion}

The MG-RAST analysis showed that the analyzed starter sample had an alpha-diversity of 68.84 species. Taxonomic hit distribution of the sample at domain level showed that $39.2 \%$ sequences belonged to Bacteria and $60.4 \%$ sequences belonged to Eukaryota. Taxonomic hits of distribution at phylum level showed that $38.7 \%$ sequences belonged to Streptophyta, $23 \%$ sequences belonged to Proteobacteria, and $15.8 \%$ belonged to Ascomycota. According to the MEGAN analysis, the total 2,78,231 sequences were found to have maximum abundance of Rhizopus delemar followed by Mucor circinelloides, Lactobacillus plantarum, Meyerozyma gulliermondii, and so on. Along with some efficient molds and yeasts, a wide range of environmental (opportunistic) contaminants were also found, which may pose serious health hazards. At this point, we assume that the main difference between traditional and industrial starters is that traditional starters have a higher 
resilience over an industrial one. Therefore, it may be suggested that a study on changes of microbiota during spontaneous fermentation at different time points would throw more light into the role of the various microorganisms.

Acknowledgments This study was funded by the National Agricultural Innovation Project, Component-IV (under Indian Council of Agricultural Research). The authors are grateful to all the faculty and staff members of the Department of Agricultural Biotechnology and DBT-AAU Centre, Assam Agricultural University, Jorhat (India).

\section{Compliance with ethical standards}

Conflict of interest The authors declare that they have no conflict of interest.

Open Access This article is distributed under the terms of the Creative Commons Attribution 4.0 International License (http:// creativecommons.org/licenses/by/4.0/), which permits unrestricted use, distribution, and reproduction in any medium, provided you give appropriate credit to the original author(s) and the source, provide a link to the Creative Commons license, and indicate if changes were made.

\section{References}

AOAC (1970) official methods of analysis, 10th edn., Association of Official Agricultural Chemist, Washington DC

Balasubramaniam T, Sadasivam S (1987) Changes in starch, oil, protein and amino acids in developing seeds of Okra (Abelomoschus esculentus L. Moench). Plant Foods Hum Nutr 37:41-46

Barnett JA, Payne RW, Yarrow D (2000) Yeasts: characteristics and identification, 3rd edn. Cambridge University Press, Cambridge

Baronian KHR (2004) The use of yeasts and moulds as sensing elements in biosensors. Biosens Bioelec 19:953-962

Battcock M, Ali SA (1993) Fermented fruits and vegetables. FAO Agricultural Services Bulletin 134:13-28

Bokulich NA, Bamforth CW, Mills DA (2012) Brewhouse-resident microbiota are responsible for multi-stage fermentation of American coolship ale. PLoS One 7:e35507

Breuer U, Harms H (2006) Debaryomyces hansenii-an extremophilic yeast with biotechnological potential. Yeast 23:415-437

Campanaro S, Treu L, Vendramin V et al (2014) Metagenomic analysis of the microbial community in fermented grape marc reveals that Lactobacillus fabifermentans is one of the dominant species: insights into its genome structure. Appl Microbiol Biotechnol 98(13):6015-6037

Chan XY, Chua KH, Puthucheary SD et al (2012) Draft genome sequence of an Aeromonas sp. strain 159 clinical isolate that shows quorum-sensing activity. J Bacteriol 194:6350

Chatonnet P, Dubourdieu D, Boidron JN, Pons M (1992) The origin of ethylphenols in wines. J Sci Food Agric 60:165-178

Chatonnet P, Dubourdieu D, Boidron JN (1995) The influence of Brettanomyces-Dekkera sp yeasts and lactic acid bacteria on the ethylphenol content of red wines. Am J Enol Vitic 46:463-468

Cocolin L, Rantsiou K, Iacumin L, Zironi R, Comi G (2004) Molecular detection and identification of Brettanomyces/Dekkera bruxellensis and Brettanomyces/Dekkera anomalus in spoiled wines. Appl Environ Microbiol 70:1347-1355

Crabb WD (1999) Commodity scale production of sugars from starches. Curr Opin Microbiol 2:252-256

Das AJ, Deka SC, Miyaji T (2012) Methodology of rice beer preparation and various plant materials used in starter culture preparation by some tribal communities of North-East India: a survey. Int Food Res J 19:101-107

Davenport RR (1980) Cold-tolerant yeasts and yeast-like organisms. In: Skinner FA, Passmore SM, Davenport RR (eds) Biology and activities of yeasts. Academic Press, London, pp 215-230

De Mot R, Van Dijck K, Donkers A, Verachtert H (1985) Potentialities and limitations of direct alcoholic fermentation of starchy material with amylolytic yeasts. Appl Microbiol Bitechnol 22:222-226

Dijkshoorn L, Nemec A, Seifert H (2007) An increasing threat in hospitals: multidrug-resistant Acinetobacter baumannii. Nat Rev Microbiol 5:939-951

Droby S, Chalutz E, Wilson CL, Wisniewski M (1989) Characterization of the biocontrol activity of Debaryomyces hansenii in the control of Penicillium digitatum on grapefruit. Can J Microbiol 35:794-800

Dung NTP, Rombouts FM, Nout MJR (2005) Development of defined mixed-culture fungal fermentation starter granule for controlled production of rice wine. Innov Food Sci Emerg J 6:429-441

Edlin DAN, Narbad A, Dickinson JR, Lloyd D (1995) The biotransformation of simple phenolic compounds by Brettanomyces anomalus. FEMS Microbiol Lett 125:311-316

Ellis JJ (1985) Species and varieties in the Rhizopus arrhizusRhizopus oryzae group as indicated by their DNA complementarity. Mycologia 77:243-247

Ercolini D (2004) PCR-DGGE fingerprinting: novel strategies for detection of microbes in food. J Microbiol Methods 56:297-314

Fleet GH (1993) Wine-microbiology and biotechnology. Harwood Academic Publishers, Chur

Fungelsang K (1997) Wine microbiology. The Chapman \& Hall Enology Library, New York

Gandjar I (1999) Fermented foods-fermentations of the Far East. In: Robinson RK, Batt CA, Patel PD (eds) Encyclopedia of food microbiology. Academic Press, London, pp 767-773

Gilbert JA, Field D, Huang Y et al (2008) Detection of large numbers of novel sequences in the metatranscriptomes of complex marine microbial communities. PLoS One 3:e3042

Haard NF, Odunfa SA, Lee CH et al (1999) Fermented cereals a global perspective. FAO Agricultural Services Bulletin 138:97

Hanai S (1992) Odo ni Umareta Sake: Chugokushu sono Gijyutsu to Rekishi [Alcoholic Beverages Originating in the Yellow River Basin] Toho Shoten, Tokyo

Haruta S, Ueno S, Egawa I et al (2006) Succession of bacterial and fungal communities during a traditional pot fermentation of rice vinegar assessed by PCR-mediated denaturing gradient gel electrophoresis. Int J Food Microbiol 109:79-87

Hesseltine CW (1983) Microbiology of oriental fermented foods. Annu Rev Microbiol 37:575-601

Hesseltine CW, Rogers R, Winarno FG (1988) Microbiological studies on amylolytic oriental fermentation starters. Mycopathol 101:141-155

Hong X, Chen J, Liu L et al (2016) Metagenomic sequencing reveals the relationship between microbiota composition and quality of Chinese Rice Wine. Sci Rep 6:26621

Huang Y, Buska PK, Grella MN et al (2014) Identification of a $\beta$ glucosidase from the Mucor circinelloides genome by peptide pattern recognition. Enzyme Microb Tech 67:47-52

Huson DH, Auch AF, Qi J, Schuster SC (2007) MEGAN analysis of metagenomic data. Genome Res 17:377-386

Iwen PC, Sigler L, Noel RK, Freifeld AG (2007) Mucor circinelloides was identified by molecular methods as a cause of primary cutaneous zygomycosis. J Clin Microbiol 45:636-640

Jazwiak KP, Rozmierska J, Chablowska B et al (2013) Starter cultures for lactic acid fermentation of sweet pepper, patty-pan squash and tomatoes. Polish J Food Nutr Sci 63:95-102

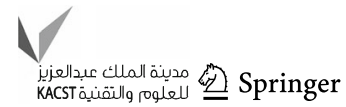


Jeyarama K, Mohendro Singh W, Capece A, Romano P (2008) Molecular identification of yeast species associated with 'Hamei' - a traditional starter used for rice wine production in Manipur, India. Int J Food Microbiol 124:115-125

Jung JY, Lee SH, Kim JM et al (2011) Metagenomic analysis of kimchi, a traditional Korean fermented food. Appl Environ Microbiol 77(7):2264-2274

Khan ZU, Ahmad S, Brazda A, Chandy R (2009) Mucor circinelloides as a cause of invasive maxillofacial zygomycosis: an emerging dimorphic pathogen with reduced susceptibility to posaconazole. J Clin Microbiol 47:1244-1248

Laluce C, Mattoon JR (1984) Development of rapidly fermenting strains of Saccharomyces diastaticus for direct conversion of starch and dextrin to ethanol. Appl Environ Microbiol 48:17-25

Lazarevic V, Whiteson K, Huse S et al (2009) Metagenomic study of the oral microbiota by Illumina high-throughput sequencing. J Microbiol Methods 79:266-271

Lee AC, Fujio Y (1999) Microflora of banh men, a fermentation starter from Vietnam. World J Microbiol Biotechnol 15:51-55

Lim G (1991) Indigenous fermented foods in South East Asia. ASEAN Food J 6:83-101

Limtong S, Sintara S, Suwannarit P, Lotong N (2002) Yeast diversity in Thai traditional alcoholic starter (Loog-pang). Kasetsart J Nat Sci 36:149-158

Limtong S, Sintara S, Suwanarit P, Lotong N (2005) Species diversity of molds in Thai traditional fermentation starters (Loog-Pang). Kasetsart J Nat Sci 39:511-518

Lv XC, Huang XL, Zhang W et al (2013) Yeast diversity of traditional alcohol fermentation starters for Hong Qu glutinous rice wine brewing, revealed by culture dependent and cultureindependent methods. Food Control 34:183-190

Mao AA, Odyuo N (2007) Traditional fermented foods of the Naga tribes of Northeastern India. Indian J Tradit Know 6:37-41

Masoud W, Takamiya M, Vogensen FK et al (2011) Characterization of bacterial populations in Danish raw milk cheeses made with different starter cultures by denaturing gradient gel electrophoresis and pyrosequencing. Int Dairy J 21:142-148

Meroth CB, Hammes WP, Hertel C (2003) Identification and population dynamics of yeasts in sourdough fermentation processes by PCR-denaturing gradient gel electrophoresis. Appl Environ Microbiol 69:7453-7461

Meyer F, Paarmann D, D'Souza M et al (2008) The metagenomics RAST server-a public resource for the automatic phylogenetic and functional analysis of metagenomes. BMC Bioinformatics 9:386

Nalbantoglu U, Cakar A, Dogan H et al (2014) Metagenomic analysis of the microbial community in kefir grains. Food Microbiol 41:42-51

Nanni V, Zanetti M, Bellucci M et al (2013) The peach (Prunus persica) defensin PpDFN1 displays antifungal activity through specific interactions with the membrane lipids. Plant Pathol 62:393-403

Norkrans B (1966) Studies on marine occurring yeasts: growth related to $\mathrm{pH}, \mathrm{NaCl}$ concentration and temperature. Arch Microbiol 54:374-392

Onishi H (1963) Osmophilic yeasts. Adv Food Res 12:53-94

Paster N, Drody S, Chalutz E et al (1993) Evaluation of the potential of the yeast Pichia guilliermondii as a biological control agent against Aspergillus flavus and fungi of stored soya beans. Mycol Res 97:1201-1206

Peleg AY, Seifert H, Paterson DL (2008) Acinetobacter baumannii: emergence of a successful pathogen. Clin Microbiol Rev 21:538-582

Petersson S, Schnürer J (1995) Biocontrol of mold growth in high moisture wheat stored under airtight conditions by Pichia anomala, Pichia guilliermondii, and Saccharomyces cerevisiae. Appl Environ Microbiol 61:1027-1032

Phuc NH (1998) The fermentation processes of traditional foods. Agriculture Publisher, HCM City

Prakitchaiwattana CJ, Fleet GH, Heard GM (2004) Application and evaluation of denaturing gradient gel electrophoresis to analyse the yeast ecology of wine grapes. FEMS Yeast Res $4: 865-877$

Qin J, Li R, Raes J, Arumugam M, Burgdorf KS et al (2010) A human gut microbial gene catalogue established by metagenomic sequencing. Nature 464:59-65

Quigley L, O'Sullivan O, Beresford TP et al (2012) High-throughput sequencing for detection of subpopulations of bacteria not previously associated with artisanal cheeses. Appl Environ Microbiol 78:5717-5723

Rantsiou K, Urso R, Iacumin L et al (2005) Culture dependent and independent methods to investigate the microbial ecology of Italian fermented sausages. Appl Env Microbiol 71:1977-1986

Ratledge C, Tan KH (1990) Oils and fats: production degradation and utilization by yeasts. In: Verachtert H, De Mot R (eds) Yeast biotechnology and biocatalysis. Marcel Dekker, New York, pp 223-254

Reid G (1999) The scientific basis for probiotic strains of Lactobacillus. Appl Environ Microbiol 65:3763-3766

Rong RJ, Li ZM, Wang DL et al (2009) Research progress on microorganisms in Chinese liquor Qu. China Brewing 6:5-8 (In Chinese)

Sadasivam S, Manickam A (1996) Biochemical methods, 2nd edn. New Age International Pubishers, New Delhi

Sarma HK (2002) Isolation and characterization of yeast strains from indigenous starter culture for alcoholic fermentation. In: Tiwari SC, Sharma GD (eds) Microbial diversity: Status and potential applications. Scientific Book Centre, Guwahati, pp 85-97

Schipper MAA (1984) A revision of the genus Rhizopus I The Rhizopus stolonifer-group and Rhizopus oryzae Studies. Mycol (Baarn) 25:1-19

Schipper MAA, Stalpers JA (1984) A revision of the genus Rhizopus II The Rhizopus microsporus-group Studies. Mycol (Baarn) 25:20-34

Seesuriyachan P (2011) Effect of spices and herbs on the growth of Pichia burtonii DA 69, and Saccharomyces cerevisiae Biot 88 using the application of Plackett-Burman screening design. Chiang Mai J Sci 38:151-155

Shi JH, Xiao YP, Li XR et al (2009) Analyses of microbial consortia in the starter of Fen liquor. Lett Appl Microbiol 48:478-485

Soccol C, Marin B, Raimbault M, Lebeault JM (1994) Breeding and growth of Rhizopus in raw cassava by solid state fermentation. Appl Microbiol Biotechnol 41:330-336

Somogyi M (1952) Notes on sugar determination. J Biol Chem 195:19-23

Steinkraus KH (1989) Industrialization of indigenous fermented foods, Basel. Marcel Dekker Inc, New York

Tamang JP, Sarkar PK (1995) Microflora of Murcha: an amylolytic fermentation starter. Microbios 81(327):115-122

Tanti B, Gurung L, Sarma HK, Buragohain AK (2010) Ethnobotany of starter cultures used in alcohol fermentation by a few ethnic tribes of Northeast India. Indian $\mathbf{J}$ Tradit Know 9:463-466

Thanh VN, Mai LT, Tuan DA (2008) Microbial diversity of traditional Vietnamese alcohol fermentation starters (banh men) as determined by PCR-mediated DGGE. Int J Food Microbiol 128:268-273

Tilbury RH (1980) Xerotolerant (osmophilic) yeasts. In: Skinner FA, Passmore SM, Davenport RR (eds) Biology and activities of yeasts. Academic Press, London, pp 153-179 
Upadhyaya ML, Gupta RC (2009) Hyperparasitism among some dominant leaf surface fungi of Setaria italica (Linn) Beauv. Indian Phytopath 62:115-116

Urich T, Lanzen A, Qi J, Huson DH et al (2008) Simultaneous assessment of soil microbial community structure and function through analysis of the meta-transcriptome. PLoS One 3:e2527

Vaughan-Martini A, Martini A (1995) Facts, myths and legends on the prime industrial microorganism. Int $\mathrm{J}$ Food Microbiol 14:514-522

Weitzman I, McGough DA, Rinaldi MG, Della-Lata P (1996) Rhizopus schipperae, sp nov, a new agent of zygomycosis. Mycotaxon 59:217-225
Wisniewski M, Biles CL, Droby S et al (1991) Mode of action of postharvest biocontrol yeast, Pichia guilliermondii I: characterization of attachment to Botrytis cinerea. Physiol Mol Plant Pathol 39:245-258

Xie GF, Li WJ, Lu J et al (2007) Isolation and identification of representative fungi from shaoxing rice wine wheat $q u$ using a polyphasic approach of culture-based and molecular-based methods. J Inst Brew 113:272-279

Yemm EW, Willis AJ (1954) The estimation of carbohydrates in plant extracts by anthrone. Biochem J 57:508-514

Yuan GF, Jong SC (1984) A new obligate azygosporic species of Rhizopus. Mycotaxon 20:397-400 